Supporting Information

Spin-Flip Density Functional Theory for Redox

Properties of Organic Photoredox Catalyst in

\title{
Excited States
}

Jiyoon Choi ${ }^{+} \neq$and Hyungjun Kim ${ }^{\dagger} \neq *$

†Department of Chemistry, Incheon National University, 119

Academy-ro, Yeonsu-gu, Incheon 22012, Republic of Korea

${ }^{\ddagger}$ Research Institute of Basic Sciences, Incheon National University, 119 Academy-ro, Yeonsu-gu, Incheon 22012, Republic of Korea 
Top view

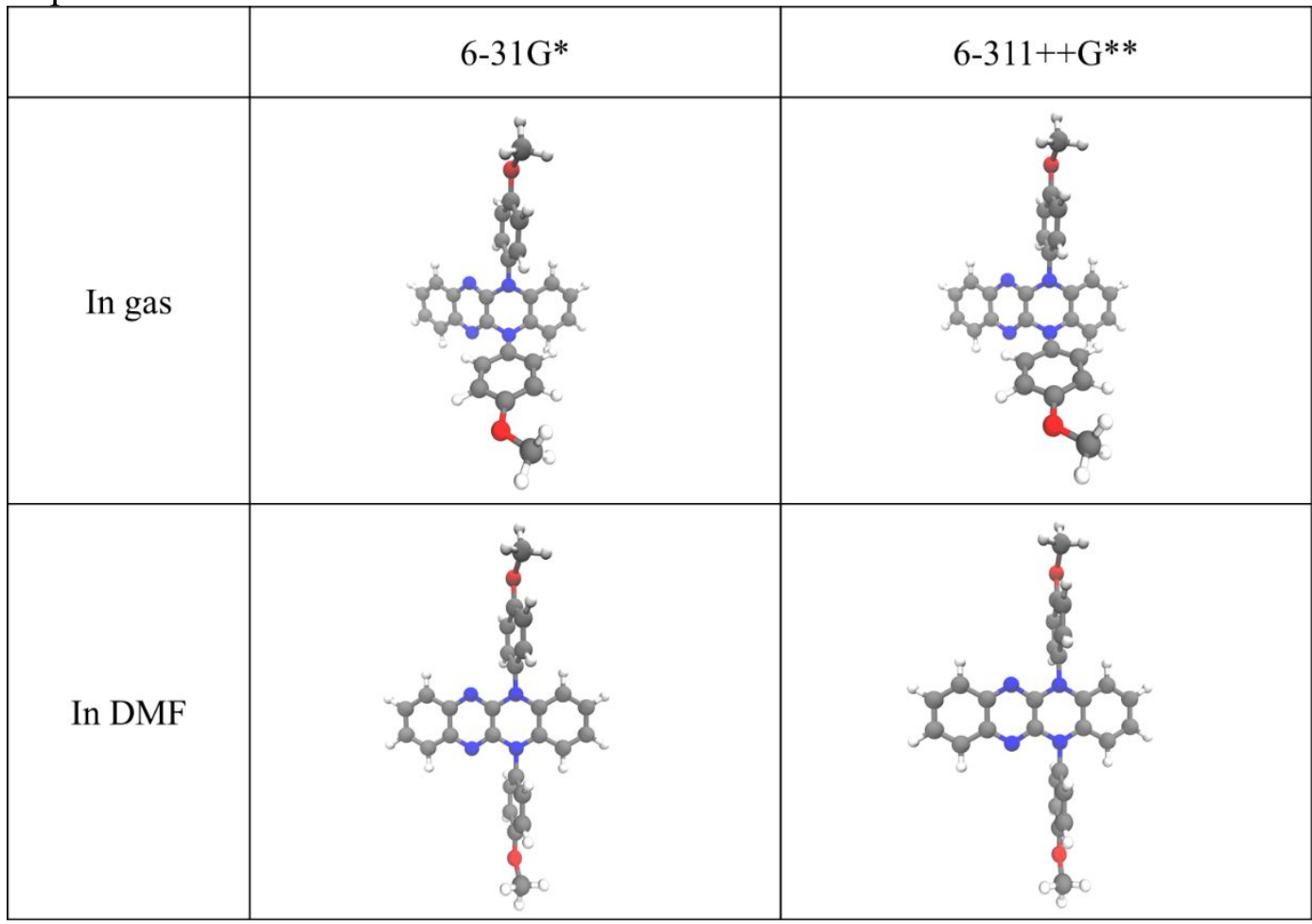

Figure S1. Top view for optimized $\mathrm{S}_{1}$ geometries of DHQQ-PMP by TD-B3LYP-D with varying dielectric environment condition and basis sets size. Color scheme: hydrogen — white, carbon - gray, nitrogen - blue, oxygen - red. 
Top view

\begin{tabular}{|l|c|c|}
\hline & 6-31G* & 6-311++G** \\
\hline In gas & & \\
\hline & & \\
\hline
\end{tabular}

Side view

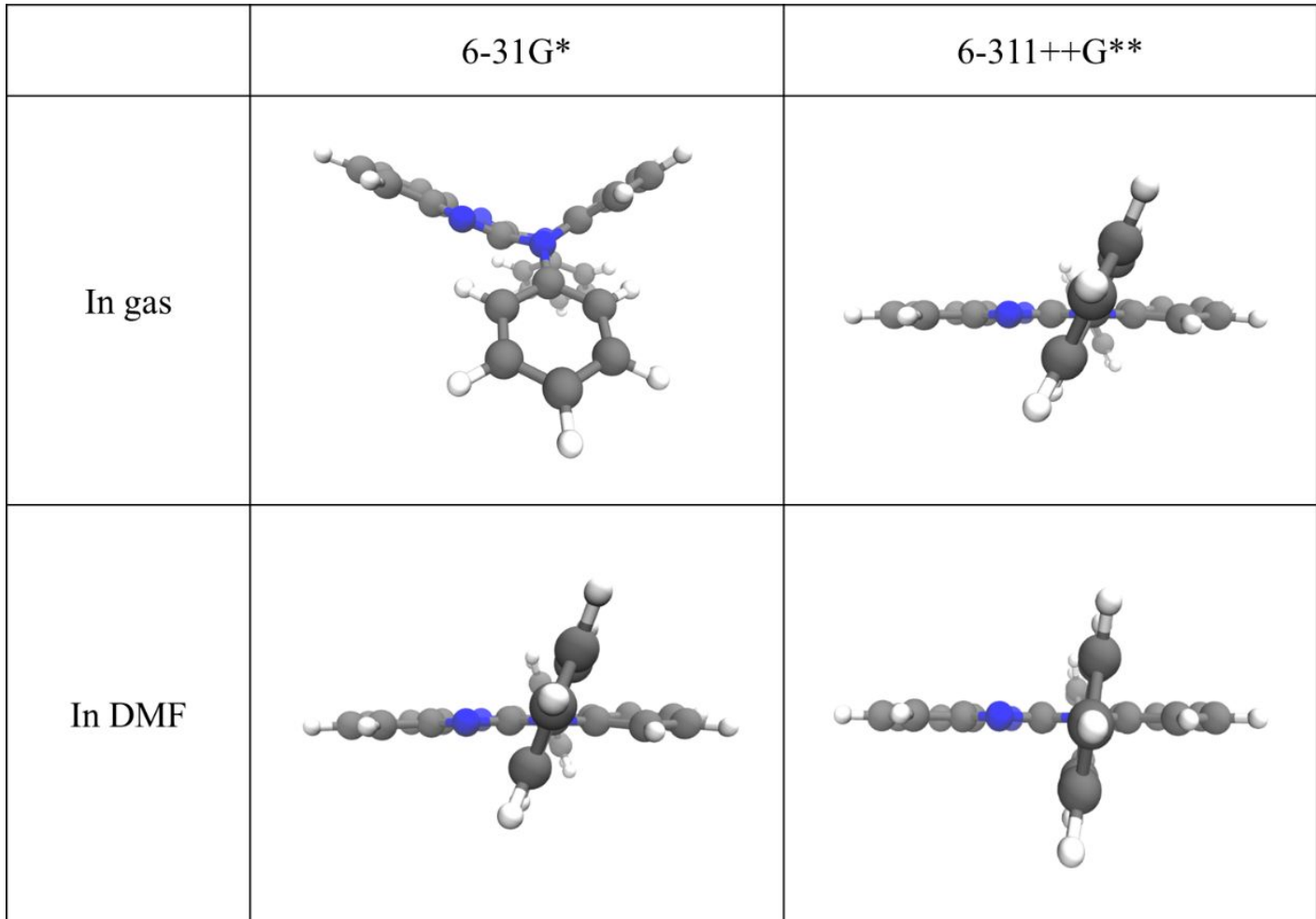

Figure S2. Top and side view for optimized $\mathrm{S}_{1}$ geometries of DHQQ-Ph by TD-B3LYP-D with varying dielectric environment condition and basis sets size. Color scheme: hydrogenwhite, carbon-gray, nitrogen—blue, oxygen — red. 
Table S1. The lowest absorption (Abs) and the lowest emission energy (Emi) calculated by TD-B3LYP/6-311++G**/SMD(DMF) based on the geometry optimized by B3LYP/6$31 \mathrm{G}^{*} /$ gas and TD-B3LYP/6-31G*/gas, respectively [eV] (Calc: Calculated value, Expt: experimental value, Diff: difference between Calc and Expt).

\begin{tabular}{|c|c|c|c|c|c|c|c|c|}
\hline & \multicolumn{3}{|c|}{ Abs } & \multicolumn{3}{c|}{ Emi } & \multicolumn{2}{c|}{ Stokes shift } \\
\hline DHQQ-CF 3 & 3.04 & 3.04 & 0.00 & 2.64 & 2.55 & 0.09 & 0.40 & 0.49 \\
& & & & & & & & \\
\hline DHQQ-Et & 3.27 & 3.01 & 0.26 & 2.80 & 2.58 & 0.22 & 0.47 & 0.43 \\
\hline DHQQ-Ph & 3.16 & 3.04 & 0.12 & 1.89 & 2.57 & -0.68 & 1.27 & 0.47 \\
& & & & & & & & \\
\hline DHQQ-PMP & 3.23 & 3.02 & 0.20 & 1.74 & 2.56 & -0.82 & 1.49 & 0.46 \\
& & & & & & & & \\
\hline
\end{tabular}

Table S2. The lowest absorption (Abs) and the lowest emission energy (Emi) calculated by TD-B3LYP/6-311++G**/SMD(DMF) based on the geometry optimized by B3LYP/6$31 \mathrm{G}^{*} / \mathrm{SMD}(\mathrm{DMF})$ and TD-B3LYP/6-31G*/SMD(DMF), respectively [eV]. Mean signed deviation (MSD) is given in the last row (Calc: Calculated value, Expt: experimental value, Diff: difference between Calc and Expt).

\begin{tabular}{|c|c|c|c|c|c|c|c|c|}
\hline & \multicolumn{3}{|c|}{ Abs } & \multicolumn{3}{|c|}{ Emi } & \multicolumn{2}{|c|}{ Stokes shift } \\
\hline & Calc & Expt & Diff & Calc & Expt & Diff & Calc & Expt \\
\hline $\mathrm{DHQQ}-\mathrm{CF}_{3}$ & 3.24 & 3.04 & 0.20 & 2.64 & 2.55 & 0.09 & 0.60 & 0.49 \\
\hline DHQQ-Et & 3.28 & 3.01 & 0.27 & 2.82 & 2.58 & 0.24 & 0.46 & 0.43 \\
\hline DHQQ-Ph & 3.26 & 3.04 & 0.12 & 2.72 & 2.57 & 0.15 & 0.54 & 0.47 \\
\hline DHQQ-PMP & 3.25 & 3.02 & 0.23 & 2.73 & 2.56 & 0.17 & 0.52 & 0.46 \\
\hline MSD & & & 0.23 & & & 0.16 & & \\
\hline
\end{tabular}




\begin{tabular}{|c|c|c|c|}
\hline DHQQ-CF & DHQQ-Et & DHQQ-Ph & DHQQ-PMP \\
\hline 30 & & & \\
\hline & & & 8 \\
\hline
\end{tabular}

Figure S3. Optimized $\mathrm{S}_{0}$ geometries of four PC investigated in this study by B3LYP-D/6$31 \mathrm{G}^{*} / \mathrm{SMD}(\mathrm{DMF})$. Color scheme: hydrogen-white, carbon-gray, nitrogen-blue, oxygen—red, fluorine - pink.

\begin{tabular}{|c|c|c|c|}
\hline DHQQ-CF & DHQQ-Et & DHQQ-Ph & DHQQ-PMP \\
\hline 30 & & & \\
\hline & &
\end{tabular}

Figure S4. Optimized $\mathrm{S}_{1}$ geometries of four PC investigated in this study by TD-B3LYP-D/631G*/SMD(DMF) Color scheme: hydrogen — white, carbon - gray, nitrogen — blue, oxygenred, fluorine - pink (The structure of DHQQ- $\mathbf{C F}_{\mathbf{3}}$ is from gas phase). 


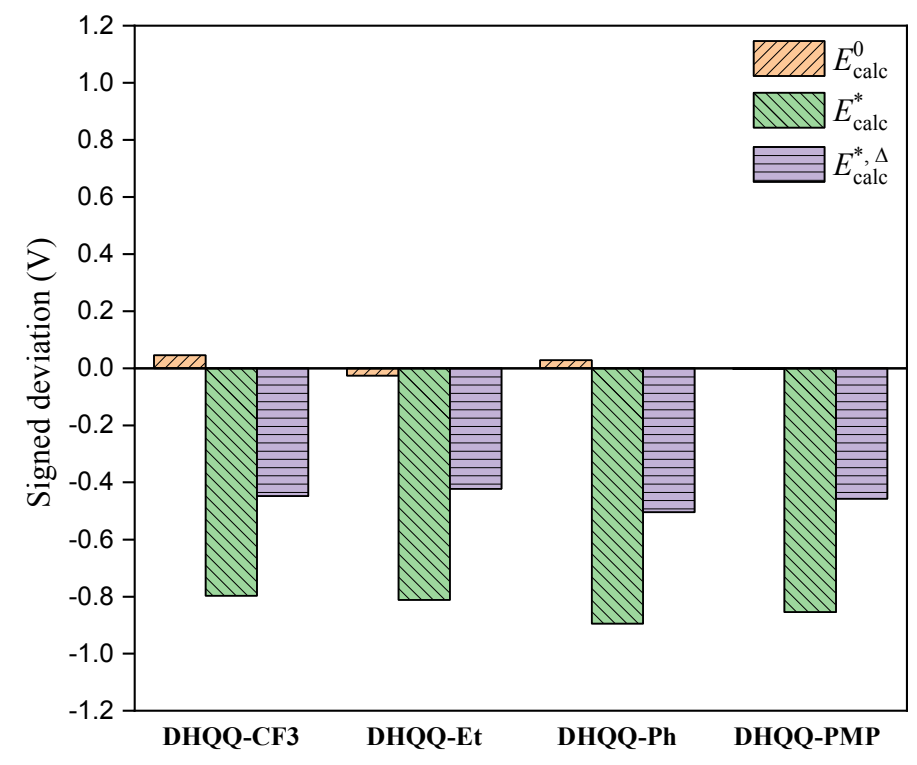

Figure S5. Signed deviation of $E_{\text {calc }}^{0}$ (orange, left diagonal line), $E_{\text {calc }}^{*}$ (green, right diagonal line), and $E_{\text {calc }}^{*} \Delta$ (purple, horizontal line) predicted by the M06-2X-D functional from $E_{\text {expt }}^{*, \Delta}$ for four PCs [V]

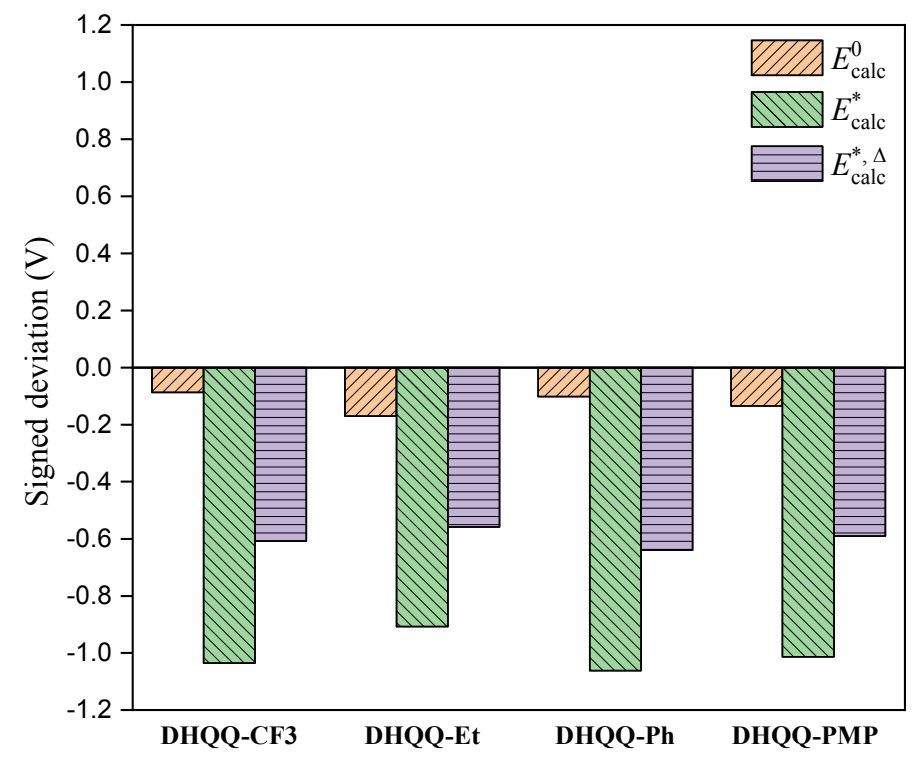

Figure S6. Signed deviation of $E_{\text {calc }}^{0}$ (orange, left diagonal line), $E_{\text {calc }}^{*}$ (green, right diagonal line), and $E_{\text {calc }}^{*}$ (purple, horizontal line) predicted by the $\omega$ B97X-D functional from $E_{\text {expt }}^{*, \Delta}$ for four PCs [V] 
Table S3. Mean signed deviation of $E_{\text {calc }}^{0}$ and $E_{\text {calc }}^{* \Delta}$ for four PCs with varying amount of HFX in B3LYP-D

\begin{tabular}{|c|c|c|}
\hline & \multicolumn{2}{|c|}{ Mean signed deviation } \\
\hline HFX (\%) & $E_{\text {calc }}^{0}$ & $E_{\text {calc }}^{*}$ \\
\hline 0 & -0.38 & -0.27 \\
\hline 20 & -0.28 & -0.43 \\
\hline 28 & -0.24 & -0.49 \\
\hline 40 & -0.17 & -0.58 \\
\hline 50 & -0.12 & -0.66 \\
\hline
\end{tabular}

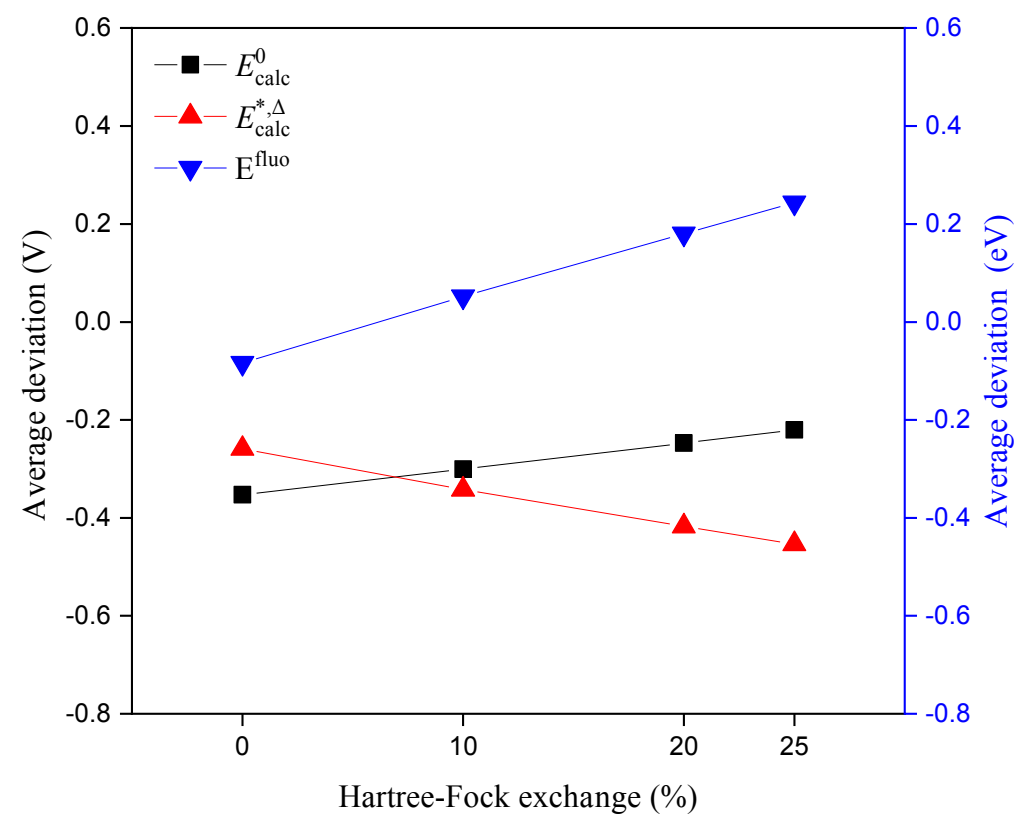

Figure S7. Mean signed deviation of $E_{\text {calc }}^{0}$ (black square), $E_{\text {calc }}^{*}$ (red upper triangles), and $E^{\text {fluo }}$ (blue lower triangles) from the experiments with varying amount of HFX in PBE0-D [Units: $\mathrm{V}$ for $E_{\text {calc }}^{0}$ and $E_{\text {calc }}^{*}$, and $\mathrm{eV}$ for $E^{\text {fluo }}$ ]. 


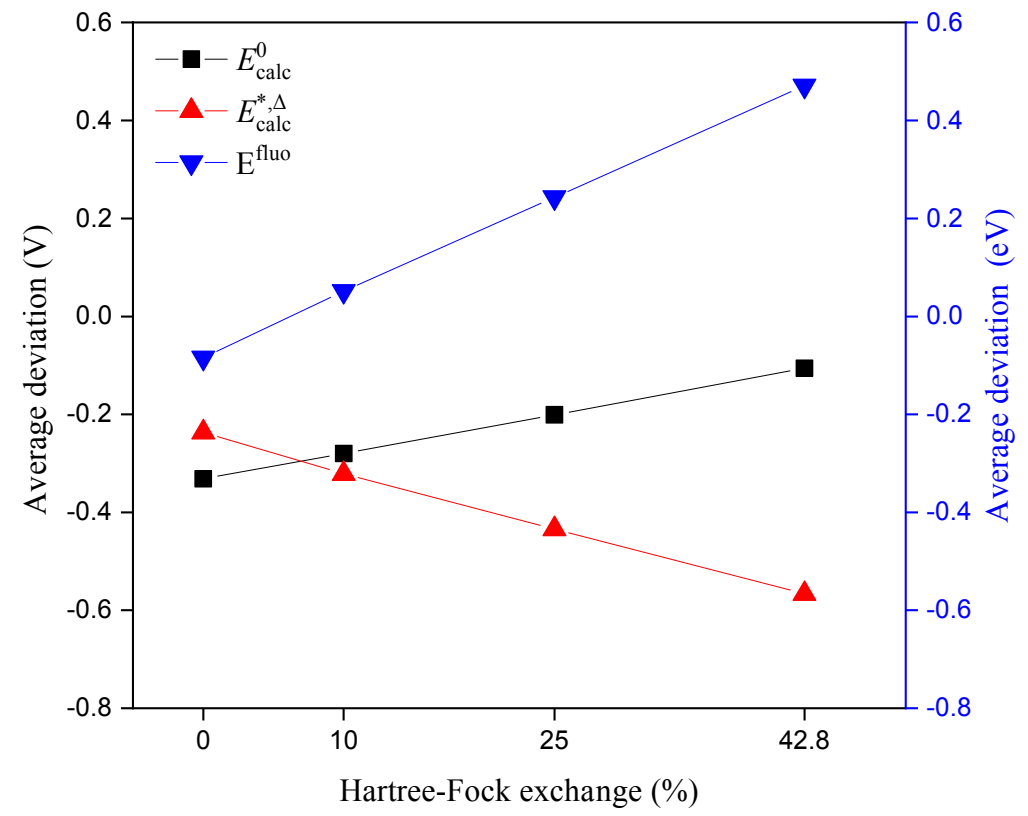

Figure S8. Mean signed deviation of $E_{\text {calc }}^{0}$ (black square), $E_{\text {calc }}^{*}$ (red upper triangles), and $E^{\text {fluo }}$ (blue lower triangles) from the experiments with varying amount of HFX in MPW1K-D [Units: $\mathrm{V}$ for $E_{\text {calc }}^{0}$ and $E_{\text {calc }}^{*}$, and $\mathrm{eV}$ for $E^{\text {fluo }}$ ].

Table S4. Hole size $(\AA)$ computed by TD-B3LYP-D and its derivatives with varying amount of HFX

\begin{tabular}{|c|c|c|c|c|c|}
\hline HFX (\%) & 0 & 20 & 28 & 40 & 50 \\
\hline DHQQ-CF $_{3}$ & 3.24516 & 3.214360 & 3.206348 & 3.200315 & 3.195736 \\
\hline DHQQ-Et & 3.289974 & 3.246455 & 3.231766 & 3.215086 & 3.202902 \\
\hline DHQQ-Ph & 3.285722 & 3.240500 & 3.225241 & 3.210461 & 3.199785 \\
\hline DHQQ-PMP & 3.545811 & 3.320186 & 3.280101 & 3.244216 & 3.223146 \\
\hline
\end{tabular}

Table S5. Electron size $(\AA)$ computed by TD-B3LYP-D and its derivatives with varying amount of HFX

\begin{tabular}{|c|c|c|c|c|c|}
\hline HFX (\%) & 0 & 20 & 28 & 40 & 50 \\
\hline DHQQ-CF $_{3}$ & 4.156809 & 3.461622 & 3.257361 & 3.048172 & 2.927993 \\
\hline DHQQ-Et & 3.029489 & 2.920616 & 2.882103 & 2.831331 & 2.793406 \\
\hline DHQQ-Ph & 3.319977 & 2.979917 & 2.901684 & 2.817082 & 2.762107 \\
\hline DHQQ-PMP & 3.080841 & 2.897929 & 2.842551 & 2.776447 & 2.730533 \\
\hline
\end{tabular}




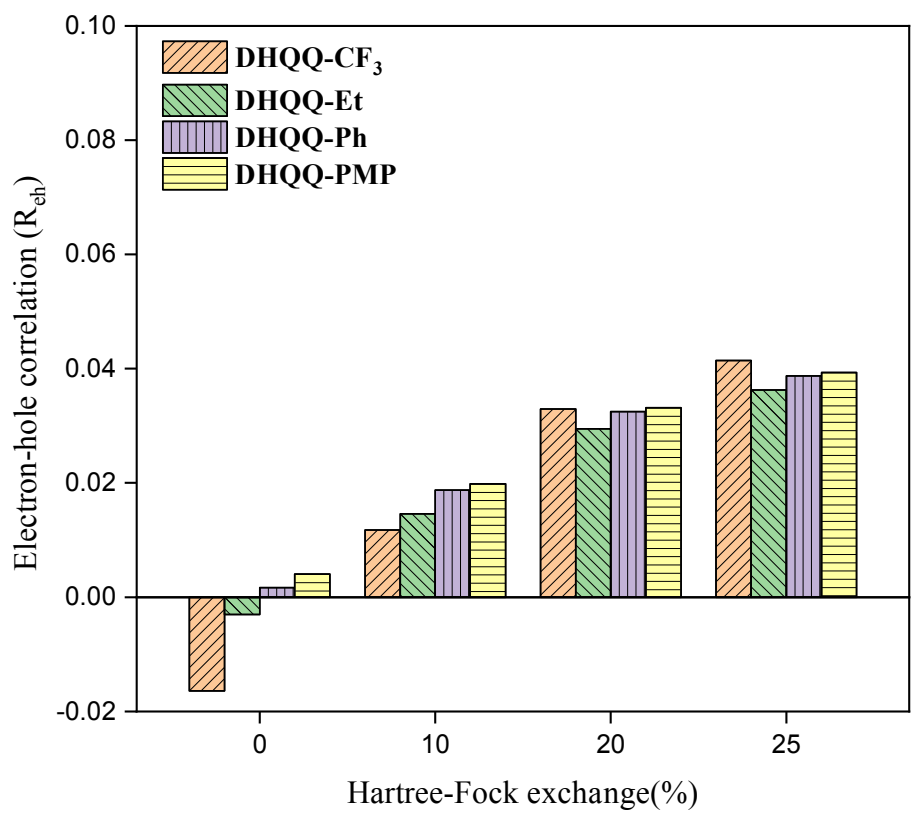

Figure S9. Electron-hole correlation $\left(R_{\mathrm{eh}}\right)$ value of $\mathbf{D H Q Q}-\mathbf{C F}_{\mathbf{3}}$ (orange, left diagonal line), DHQQ-Et (green, right diagonal line), DHQQ-Ph (purple, vertical line), and DHQQ-PMP (yellow, horizontal line) computed by TD-PBE0-D and its derivatives with varying amount of HFX

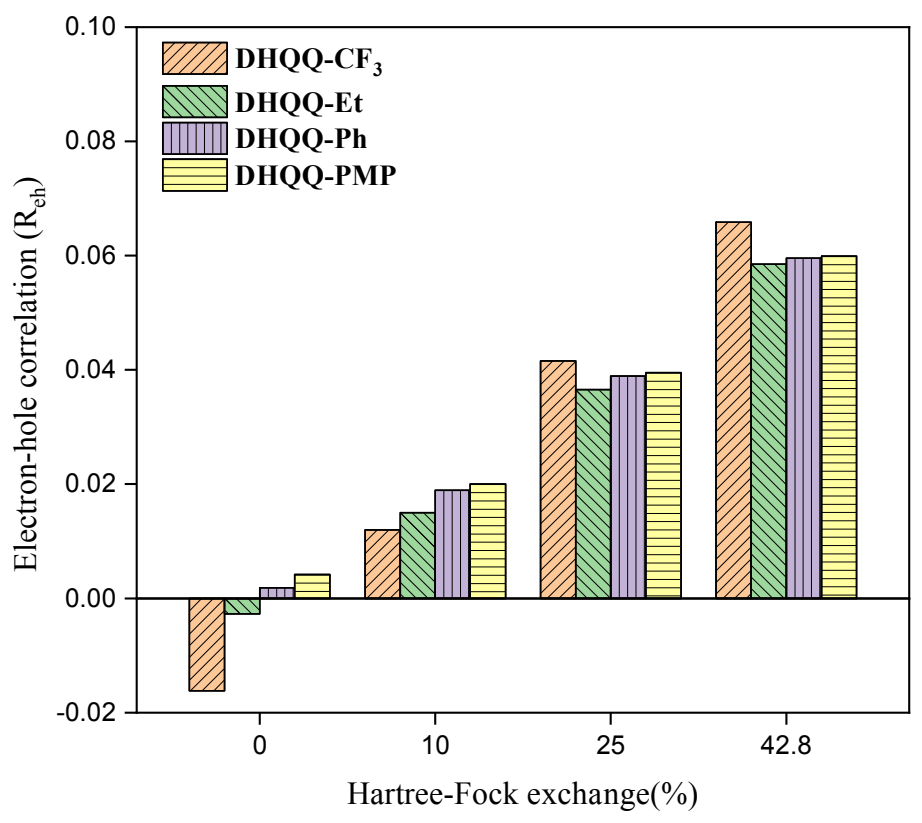

Figure S10. Electron-hole correlation $\left(R_{\mathrm{eh}}\right)$ value of $\mathbf{D H Q Q}-\mathbf{C F}_{\mathbf{3}}$ (orange, left diagonal line), DHQQ-Et (green, right diagonal line), DHQQ-Ph (purple, vertical line), and DHQQ-PMP (yellow, horizontal line) computed by TD-MPW1K-D and its derivatives with varying amount of HFX 